\title{
Consistency of Earth Rotation, Gravity, and Shape Measurements
}

\author{
Richard S. Gross* , David A. Lavallée ${ }^{\dagger}$, Geoffrey Blewitt ${ }^{\dagger}$, and Peter J. Clarke ${ }^{\dagger}$ \\ * Jet Propulsion Laboratory, California Institute of Technology \\ Mail Stop 238-600, 4800 Oak Grove Drive, Pasadena, CA 91109, USA \\ †School of Civil Engineering and Geosciences, Newcastle University \\ Cassie Building, Newcastle upon Tyne NE1 7RU, UK \\ †Nevada Bureau of Mines and Geology \& Seismological Laboratory, University of Nevada \\ Mail Stop 178, Reno, NV 89557, USA
}

\begin{abstract}
Degree-2 spherical harmonic coefficients of the surface mass density determined from independent GRACE, SLR, GPS, and Earth rotation measurements are compared to each other and to a model of the surface mass density obtained by summing the contributions of atmospheric pressure, ocean-bottom pressure, land hydrology, and a massconserving term. In general, the independent measurements are found to be quite consistent with each other and with the model, with correlations being as high as 0.87 and with the model explaining as much as $88 \%$ of the observed variance. But no measurement technique is found to be best overall. For the different degree- 2 coefficients, measurements from different techniques are found to agree best with the model. Thus, each measurement technique contributes to understanding the degree- 2 surface mass density of the Earth.
\end{abstract}

Keywords. Earth rotation, Earth shape, gravity, surface mass density

\section{Introduction}

The rearrangement of mass within the surficial fluid layers of the Earth, including the atmosphere, oceans, and water, snow and ice stored on land, causes the Earth's gravitational field to change, causes the Earth's rotation to change by changing the Earth's inertia tensor, and causes the Earth's shape to change by changing the load acting on the solid, but not rigid, Earth. Large-scale changes in the Earth's gravitational field have been measured for more than two decades by satellite tracking and more recently by the CHAMP and GRACE satellite missions. Changes in the Earth's rotation have been measured since the 1970 s by the space-geodetic techniques of satellite and lunar laser ranging (SLR and LLR) and very long baseline interferometry (VLBI) and more recently by the global positioning system (GPS). GPS can also be used to measure large-scale changes in the Earth's shape by precisely positioning the sites of a global network of ground-based GPS receivers. On time scales of months to a decade, loading of the solid Earth by surface fluids dominates the observed variations in each of these three fundamental geodetic quantities (gravity, rotation, and shape). Since these three quantities all change in response to the same changes in surface mass load, observations of these quantities must be consistent with each other.

Here we investigate temporal variations in the degree-2 spherical harmonic coefficients of the surface mass density, which corresponds to temporal variations in (1) the Earth's ellipsoidal shape, (2) the strongest harmonic coefficient of the Earth's gravitational field, and (3) the only coefficient that relates to the Earth's moment of inertia and hence to its rotation in space. Thus we choose to investigate degree- 2 coefficients because they present the best opportunity to test the consistency between three fundamentally different measurement techniques and with the largest-scale models of the Earth.

\section{Stokes Coefficients and Surface Mass Density}

The gravitational potential $U\left(\mathbf{r}_{\mathbf{0}}\right)$ of the Earth can be expanded in spherical harmonics in the usual geodetic manner as (e.g., Kaula 1966): 


$$
\begin{array}{r}
\mathrm{U}\left(\mathbf{r}_{\mathbf{o}}\right)=\frac{G M}{r_{o}} \sum_{l=0}^{\infty} \sum_{m=0}^{l}\left(\frac{a}{r_{o}}\right)^{l}\left(C_{l m} \cos m \phi_{o}\right. \\
\left.+S_{l m} \sin m \phi_{o}\right) \tilde{P}_{l m}\left(\cos \theta_{o}\right)
\end{array}
$$

where $\mathbf{r}_{\mathbf{o}}\left(r_{o}, \theta_{o}, \phi_{o}\right)$ is some external field point, $G$ is the gravitational constant, $M$ is the mass of the Earth, $a$ is some reference radius that is less than $r_{o}$ and which is taken here to be the radius of the Earth, and the $\tilde{P}_{m}$ are the $4 \pi$-normalized associated Legendre functions of degree $l$ and order $m$. The dimensionless expansion coefficients $C_{l m}$ and $S_{l m}$ are known as Stokes coefficients and are the normalized multipole moments of the Earth's density field $\rho(\mathbf{r})$ (e.g., Chao and Gross 1987):

$$
\begin{aligned}
& C_{l m}+i S_{l m}= \\
& \left(1+k_{l}{ }^{\prime}\right) \frac{K_{l m}}{M a^{l}} \int_{V_{o}} r^{l} Y_{l m}(\theta, \phi) \rho(\mathbf{r}) d V
\end{aligned}
$$

where the integral extends over the volume $V_{o}$ of the Earth, the $Y_{l m}$ are the fully normalized surface spherical harmonic functions, the $k_{l}^{\prime}$ are the load Love numbers of degree $l$, and the $K_{l m}$ are normalization factors

$$
K_{l m}=(-1)^{m} \frac{2}{2 l+1} \sqrt{\left(2-\delta_{m 0}\right) \pi}
$$

From equation (2), changes in the Stokes coefficients caused by changes in density that are concentrated in an infinitesimally thin layer at the surface of the Earth can be written in terms of changes in the surface mass density $\Delta \sigma(\theta, \phi)$ as:

$$
\begin{aligned}
& \Delta C_{l m}+i \Delta S_{l m}= \\
& \left(1+k_{l}{ }^{\prime}\right) K_{l m} \frac{a^{2}}{M} \int_{\Omega_{o}} Y_{l m}(\theta, \phi) \Delta \sigma(\theta, \phi) d \Omega
\end{aligned}
$$

where the integral extends over the surface $\Omega_{o}$ of the Earth. When the change in surface mass density is expressed as a change in the pressure $\Delta p(\theta, \phi)$ that is acting on the surface of the solid Earth by $\Delta \sigma(\theta, \phi)=\Delta p(\theta, \phi) / \mathrm{g}$, then equation (4) becomes:

$$
\begin{aligned}
& \Delta C_{l m}+i \Delta S_{l m}= \\
& \left(1+k_{l}{ }^{\prime}\right) K_{l m} \frac{a^{2}}{M g} \int_{\Omega_{o}} Y_{l m}(\theta, \phi) \Delta p(\theta, \phi) d \Omega
\end{aligned}
$$

where $g$ is the gravitational acceleration that has been assumed here to be constant over the surface of the Earth.

By expanding the surface mass density in the unnormalized spherical harmonics used by Blewitt and Clarke (2003), equation (4) can be used to relate changes in the spherical harmonic coefficients of the surface mass density $\Delta \sigma_{l m}$ to changes in the Stokes coefficients as:

$$
\Delta \sigma_{l m}=\frac{1}{1+k_{l}{ }^{\prime}} \frac{M}{a^{2}} N_{l m}\left(\Delta C_{l m}+i \Delta S_{l m}\right)
$$

where the $N_{l m}$ are normalization factors:

$$
N_{l m}=\frac{2 l+1}{4 \pi}\left[\frac{\left(2-\delta_{m 0}\right)(2 l+1)(l-m) !}{(l+m) !}\right]^{1 / 2}
$$

The above equations will be used below to infer changes in the degree- 2 spherical harmonic coefficients of the surface mass density from measured changes in the degree- 2 Stokes coefficients. They will also be used to determine the degree- 2 coefficients of the surface mass density from models of atmospheric surface pressure, ocean-bottom pressure, and continental water storage.

\section{Models of Surface Mass Density}

Models of the mass density of the surface geophysical fluids are used to help assess the consistency of the measurements. The particular models used are described in this section. The sum of these models will be compared to measurements in Section 4.

\subsection{Atmospheric Surface Pressure}

The atmospheric surface pressure series used here is from the National Centers for Environmental Prediction / National Center for Atmospheric Research (NCEP/NCAR) reanalysis project (Kalnay et al. 1996). Changes in the degree- 2 spherical harmonic coefficients of the gravitational potential caused by changes in surface pressure were computed using equation (5) by assuming an inverted barometer response of the oceans to the imposed surface pressure variations. The inverted barometer response of the oceans was computed by replacing the atmospheric pressure at each oceanic grid point with the mean atmospheric pressure calculated over the domain of the global oceans. 
Degree-2 coefficients of the surface mass density were computed from the degree- 2 coefficients of the gravitational potential using equation (6). In order to match the temporal resolution and sampling interval of the GRACE measurements, 30-day averages of the 6-hour values were first formed and then the 30-day values were linearly interpolated to the epochs of the GRACE measurements. The final values for the modeled degree- 2 variations in surface mass density caused by atmospheric surface pressure variations were obtained by detrending the interpolated values by removing just the mean and the trend that was determined from a simultaneous least-squares fit for a mean, a trend, and periodic terms having frequencies of 1 cycle per year (cpy), 2 cpy, and 3 cpy.

\subsection{Ocean-Bottom Pressure}

The ocean-bottom pressure series used here is from a data assimilating oceanic general circulation model that was run at the Jet Propulsion Laboratory (JPL) as part of their participation in the Estimating the Circulation and Climate of the Ocean (ECCO) consortium (Stammer et al. 2002). The model was forced with twice daily wind stress and daily surface buoyancy fluxes from the NCEP/NCAR reanalysis project and was constrained by in situ and satellite data including expendable bathythermograph data and altimetric measurements of sea surface height. The ECCO/JPL series designator of this model run is kf049f.

Atmospheric surface pressure was not used to force the ocean model. Instead, the response of the oceans to changes in surface pressure has been taken into account here when modeling the effects of atmospheric pressure variations (see Section 3.1).

Since the ocean model is formulated under the Boussinesq approximation it conserves volume rather than mass. Artificial mass variations caused by this approximation have been removed here by computing the effect on the bottom pressure of a spatially uniform layer added to the sea surface that has just the right fluctuating thickness to a posteriori impose mass conservation (Greatbatch 1994).

Like the atmospheric surface pressure series, changes in the degree- 2 spherical harmonic coefficients of the gravitational potential caused by changes in ocean-bottom pressure were computed using equation (5). Degree-2 coefficients of the surface mass density were computed from the degree-2 coefficients of the gravitational potential using equation (6). In order to match the temporal resolution and sampling interval of the GRACE measurements, 30-day averages of the 12-hour values were first formed and then the 30-day values were linearly interpolated to the epochs of the GRACE measurements. The final values for the modeled degree-2 variations in surface mass density caused by ocean-bottom pressure variations were obtained by detrending the interpolated values.

\subsection{Land Hydrology}

The land hydrology model used here is the Euphrates version of the Land Dynamics (LaD) model of Milly and Shmakin (2002). This model consists of gridded values of the surface mass density of snow, root-zone soil water, and groundwater given at monthly intervals from January 1980 to May 2005. The sum of the individual snow, soil water, and groundwater mass densities is used here.

Changes in the degree-2 spherical harmonic coefficients of the gravitational potential caused by the total continental water storage was computed using equation (4) and degree- 2 coefficients of the surface mass density were computed from the coefficients of the gravitational potential using equation (6). The resulting monthly degree-2 surface mass density values of the continental water were linearly interpolated to the epochs of the GRACE measurements and then detrended.

\subsection{Global Surface Mass Conservation}

Since mass can neither be created nor destroyed, the total mass of the atmosphere, oceans, and continental water must be a constant. Of course, the mass of an individual component of the surface geophysical fluids like the atmosphere can change as water in its various phases cycles through it. But the total mass of all components must be a constant. The atmosphere and land hydrology models used here capture the mass changes in these surface fluids, but the ocean model does not. The mass of the ocean model used here does not change because it is not forced by any mechanism that would cause its mass to change. So global mass conservation has been imposed here by adding a layer of water to the surface of the ocean model of just the right time varying thickness to make the total mass of the atmosphere, oceans, and water stored on land a constant.

Since the surface mass density of the land hydrology model used here was given at monthly in- 
tervals, the mass-conserving ocean layer was computed at monthly intervals. Changes in the degree-2 spherical harmonic coefficients of the gravitational potential caused by the mass-conserving layer were computed using equation (4) and degree-2 coefficients of the surface mass density were computed from the coefficients of the gravitational potential using equation (6). The resulting monthly degree- 2 surface mass density values of the mass-conserving layer were linearly interpolated to the epochs of the GRACE measurements and then detrended.

\section{Consistency of Measurements}

\subsection{GRACE}

Temporal variations in the Earth's global gravitational field have been measured from space by the Gravity Recovery and Climate Experiment (GRACE) since the spacecraft were launched in March 2002 (Tapley et al. 2004). Spherical harmonic coefficients of the gravitational potential are being determined from the GRACE observations by each of the three centers of the GRACE Science Data System located at the University of Texas at Austin Center for Space Research (UTCSR), the GeoForschungsZentrum Potsdam, and the Jet Propulsion Laboratory. The release-1 (RL01) and release-4 (RL04) coefficients determined by UTCSR have been used here.

The atmosphere/ocean de-aliasing product has been added back to the degree- 2 coefficients in order to recover the full gravitational signal. While UTCSR removed the effects of the ocean pole tide from the RL04 coefficients (Bettadpur 2007), they did not remove them from the RL01 coefficients. So they were removed here using the self-consistent equilibrium ocean pole tide model of Desai (2002).

Since the UTCSR RL04 coefficients were sometimes given at slightly different epochs than the RL01 coefficients, the RL04 coefficients were linearly interpolated to the epochs of the RL01 coefficients. Degree-2 coefficients of the surface mass density were then computed from the degree-2 gravitational field coefficients using equation (6). In order to match the duration of the GPS-derived surface mass density series, the GRACE series were truncated at May 2005. The 34 monthly coefficients spanning April 2002 to May 2005 were then detrended.

Figure 1 displays the resulting degree- 2 coefficients of the surface mass density as measured by GRACE, with the UTCSR RL01 coefficients being given by the open circles, and the RL04 coefficients being given by the filled circles. Also shown as a thick solid line is the sum of the models of the degree-2 mass coefficients due to atmospheric surface pressure, ocean-bottom pressure, land hydrology, and the global mass-conserving ocean layer. Figure 2 gives the correlations between the observed and modeled series, as well as the percentage of the observed variance explained by the modeled series.

With a correlation of 0.83 , the $(2,0)$ cosine coefficients of the two GRACE solutions are significantly correlated with each other. And while the $(2,1)$ and $(2,2)$ sine coefficients are quite similar to each other, having correlations of 0.81 and 0.95 , respectively, there is less agreement between the cosine coefficients, with the correlations being 0.40 and 0.55 between the $(2,1)$ and $(2,2)$ cosine coefficients, respectively. The $(2,1)$ and $(2,2)$ sine coefficients also agree much better with the models than do the cosine coefficients. For example, the models explain $58.9 \%$ of the observed variance of the RL04 $(2,1)$ sine coefficient and have a correlation of 0.78 with it, but have a correlation of only 0.26 with the RL04 $(2,1)$ cosine coefficient and the variance actually increases when the models are removed. The RL04 $(2,0)$ coefficient agrees only slightly better with the models than the RL01 coefficient.

\subsection{Satellite Laser Ranging}

Temporal variations in the low-degree spherical harmonic coefficients of the Earth's gravitational field have been determined from laser ranging measurements to the Lageos-1 satellite since it was launched in May 1976 (e.g., Cheng and Tapley 2004). Because the zonal degree- 2 gravitational field coefficient determined in early GRACE solutions was less accurate than that determined by satellite laser ranging (SLR), and since many users of the early GRACE solutions either removed the zonal degree- 2 coefficient or replaced it with an SLR-based coefficient, the GRACE project started providing replacement values of the zonal degree-2 coefficient based upon SLR measurements (Cheng and Ries 2007). The replacement values are determined from ranges to five geodetic satellites (Lageos-1, Lageos-2, Starlette, Stella, and Ajisai) using procedures and background models consistent with those used to determine the UTCSR RL01 coefficients and are therefore meant to replace the zonal degree- 2 coefficient of just this solution. The degree-2 gravitational field coefficients from SLR 


\section{Degree-2 Coefficients of Surface Mass Density}

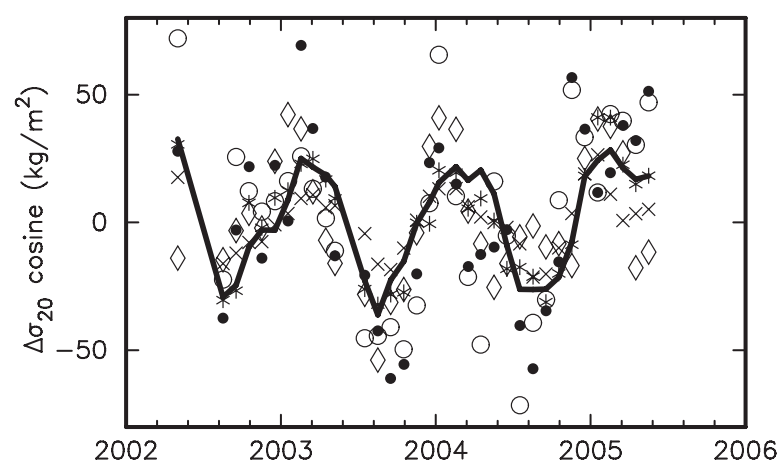

○०० GRACE (UTCSR RL01)

.. GRACE (UTCSR RL04)

*** SLR measurement

$x \times x$ GPS measurement

$\diamond \diamond \diamond$ EOP measurement

- Models
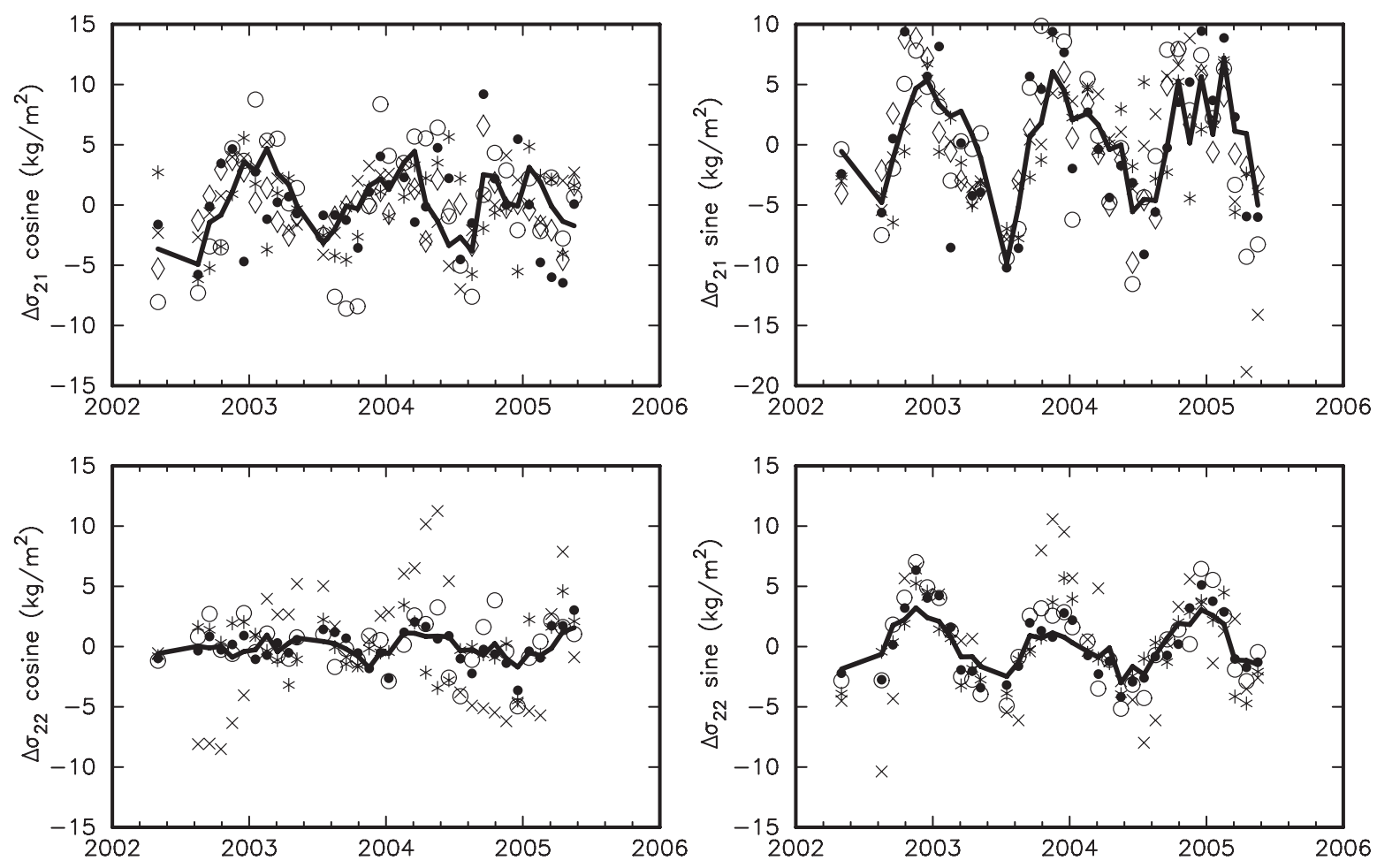

Fig. 1. Observed and modeled degree-2 spherical harmonic coefficients of the surface mass density. The observed coefficients are from the GRACE UTCSR RL01 solution (open circles), the GRACE UTCSR RL04 solution (filled circles), SLR (stars), GPS (crosses), and Earth rotation (diamonds). The modeled coefficients (solid line) are the sum of those due to atmospheric surface pressure, ocean-bottom pressure, land hydrology, and the global mass-conserving ocean layer. A mean and a trend have been removed from each of the displayed series.

used here are these replacement values, with the non-zonal coefficients provided to us by Cheng (personal communication, 2007) and coming from the same SLR solution as the zonal coefficient.

Since the SLR series used here is meant to replace the degree- 2 coefficients of the UTCSR RL01 GRACE solution, it was pre-processed in the same manner as the UTCSR RL01 series. The atmos- phere/ocean de-aliasing product was added back to the degree- 2 coefficients in order to recover the full gravitational signal and the effects of the ocean pole tide were removed using the self-consistent equilibrium ocean pole tide model of Desai (2002). Since the SLR coefficients were sometimes given at slightly different epochs than the RL01 coefficients, the SLR coefficients were linearly interpolated to 


\section{Degree-2 Coefficients of Surface Mass Density \\ (95\% significance level of correlation is 0.51 )}

\begin{tabular}{|c|c|c|c|c|c|c|}
\hline & \multicolumn{6}{|c|}{$(2,0)$ cosine } \\
\hline & Models & RLO1 & RLO4 & $S L R$ & GPS & $E O P$ \\
\hline Models & 1.0 & 0.62 & 0.70 & 0.94 & 0.88 & 0.57 \\
\hline RL01 & (37.9) & 1.0 & 0.83 & 0.65 & 0.61 & 0.47 \\
\hline RLO4 & $(48.5)$ & & 1.0 & 0.73 & 0.71 & 0.53 \\
\hline$S L R$ & $(\mathbf{8 8 . 3})$ & & & 1.0 & 0.87 & 0.68 \\
\hline GPS & (6.1) & & & & 1.0 & 0.60 \\
\hline$E O P$ & $(25.7)$ & & & & & 1.0 \\
\hline
\end{tabular}

$(2,1)$ cosine

\begin{tabular}{|c|c|c|c|c|c|c|}
\hline & Models & RLO1 & RLO4 & $S L R$ & $G P S$ & $E O P$ \\
\hline Models & 1.0 & 0.70 & 0.26 & 0.33 & 0.65 & .46 \\
\hline RLO1 & (44.4) & 1.0 & 0.40 & 0.52 & 0.49 & 0.37 \\
\hline RLO4 & $(-14.4)$ & & 1.0 & 0.03 & 0.19 & 0.59 \\
\hline$S L R$ & $(-5.9)$ & & & 1.0 & -0.03 & 0.07 \\
\hline$G P S$ & $(38.1)$ & & & & 1.0 & 0.4 \\
\hline$E O P$ & $(-14.9)$ & & & & & $1 .($ \\
\hline
\end{tabular}

$(2,2)$ cosine

\begin{tabular}{|c|c|c|c|c|c|}
\hline & Models & RLO1 & RLO4 & SLR & GPS \\
\hline Models & 1.0 & 0.40 & 0.74 & 0.26 & 0.59 \\
\hline$R L 01$ & (16.2) & 1.0 & 0.55 & 0.34 & 0.18 \\
\hline RLO4 & (51.4) & & 1.0 & 0.45 & 0.43 \\
\hline$S L R$ & $(5.0)$ & & & 1.0 & -0.09 \\
\hline$G P S$ & (15.2) & & & & 1.0 \\
\hline
\end{tabular}

\author{
greatest correlation between independent measurements \\ greatest correlation with models
}

(variance of measurement explained by models in percent) (greatest variance explained in percent)

Fig. 2. Zero-lag correlation between the various observed degree- 2 surface mass density coefficients and with the modeled coefficients. For each coefficient, the greatest correlation between the observed and modeled series is given in bold, while that between the independently observed series is given in bold italics. Shown in parentheses is the percentage of the observed variance explained by the models, where for each coefficient the greatest variance explained is given in bold. A negative value for the variance explained is obtained when the variance increases when the modeled series is removed.

the epochs of the RL01 coefficients, and they were truncated to span the same duration as the RL01 coefficients. Degree-2 coefficients of the surface mass density were computed from the SLR degree2 gravitational field coefficients using equation (6), and were then detrended.

The SLR degree-2 surface mass density coefficients are given by the stars in Figure 1. The SLR coefficients agree similarly with the two GRACE solutions except for the $(2,1)$ cosine coefficient where it agrees much better with the RL01 solution. The SLR $(2,0)$ cosine coefficient agrees much better with the models than does either GRACE solution, with the models explaining $88.3 \%$ of the SLR variance and having a correlation with the SLR of 0.94 . But the non-zonal coefficients of the GRACE solutions generally agree better with the models than do the non-zonal SLR coefficients.

\subsection{Global Positioning System}

Following the method given by Blewitt and Clarke (2003) and using the basis functions of Clarke et al. (2007), data from the global network of GPS receivers as reanalyzed at the Scripps Institution of Oceanography (SIO) have been used here to determine changes in the low-degree and order spherical harmonic coefficients of the shape of the Earth's surface, and hence of the surface mass density that is causing the shape to change. The resulting degree-2 coefficients of the surface mass density span January 1996 to May 2005 at fortnightly intervals.

In order to match the temporal resolution and sampling interval of the GRACE measurements, monthly averages of the fortnightly GPS values were first formed and then the monthly values were linearly interpolated to the epochs of the GRACE 
measurements. The GPS degree-2 surface mass density coefficients were then detrended.

The GPS degree-2 surface mass density coefficients are given by the crosses in Figure 1. The $(2,2)$ sine and cosine coefficients from GPS have greater variability than the GRACE or SLR coefficients, but the other degree-2 coefficients have similar variability. The $(2,1)$ cosine coefficient from GPS agrees nearly as well with the models as does the GRACE RL01 coefficient.

\subsection{Earth Rotation}

The observed Earth orientation series used here is the COMB2005 combined series of Gross (2006). Prior to using Earth orientation measurements to study surface mass loads, other sources of excitation should be removed from the measurements. Solid Earth and ocean tidal effects have thus been removed from the COMB2005 excess length-of-day values using the models of Yoder et al. (1981) and Kantha et al. (1998), respectively. The effects of the termensual (9.12- and 9.13-day), fortnightly (13.63and 13.66-day), and monthly (27.55-day) ocean tides have been removed from the COMB2005 polar motion excitation series using the model of Gross et al. (1997). The effects of atmospheric winds have been removed using the wind angular momentum series (Zhou et al. 2006) obtained from the International Earth Rotation and Reference Systems Service (IERS) Special Bureau for the Atmosphere (Salstein 2003) that was computed from the wind fields of the NCEP/NCAR reanalysis project. The effects of oceanic currents have been removed using the current angular momentum series (Gross et al. 2005) obtained from the IERS Special Bureau for the Oceans (Gross 2003) that was computed from the currents of the ECCO/JPL data assimilating ocean model kf049f. And decadal-scale variations in length-of-day, caused predominantly by core-mantle interactions, have been removed by applying a highpass filter with a cutoff period of 4 years. The same highpass filter was applied to the polar motion excitation values in order to remove their decadal variations.

In order to match the temporal resolution and sampling interval of the GRACE measurements, 30day averages of the daily Earth orientation values were first formed and then the 30-day values were linearly interpolated to the epochs of the GRACE measurements. Degree-2 zonal and $(2,1)$ sine and cosine coefficients of the surface mass density were computed from the length-of-day and polar motion excitation series using equations (11-12) of Gross et al. (2004). The surface mass density coefficients were then detrended.

The degree-2 surface mass density coefficients from Earth rotation measurements are given by the diamonds in Figure 1. They are in good agreement with the other measurements and agree better with the modeled $(2,1)$ sine coefficient than the other measurements.

\section{Discussion and Summary}

Degree-2 coefficients of the surface mass density determined from GRACE, SLR, GPS, and Earth rotation measurements have been compared to each other in order to assess their consistency. Depending on the particular degree- 2 coefficient, the correlations between independent measurements range from a low of 0.03 [between the SLR and UTCSR RL04 measurements of the $(2,1)$ cosine coefficient] to a high of 0.87 [between the SLR and GPS measurements of the degree- 2 zonal coefficient]. While the correlation between the UTCSR RL01 and RL04 measurements of the $(2,2)$ sine coefficient is higher at 0.95 , these measurements are both based on GRACE observations and are therefore not independent of each other. The worst agreement between the measurements is found for the $(2,1)$ cosine and $(2,2)$ cosine coefficients where the correlations between the measurements are usually less than the $95 \%$ significance level of 0.51 .

The measurements were also compared to the sum of the models of the degree- 2 mass density coefficients of atmospheric surface pressure, oceanbottom pressure, land hydrology, and a global massconserving ocean layer. The SLR measurements were found to agree best with the modeled degree-2 zonal coefficient, the Earth rotation measurements with the modeled $(2,1)$ sine coefficient, and the GRACE measurements with the other modeled degree- 2 coefficients although the GPS measurements agree nearly as well as the GRACE measurements with the modeled $(2,1)$ cosine coefficient. Except for the $(2,1)$ cosine coefficient, the GRACE UTCSR RL04 solution was found to agree as well or better with the models than the RL01 solution.

In conclusion, we find that three fundamentally different measurement techniques (based on gravity, geometry, and rotation) give mutually consistent results that agree well with large-scale models of mass redistribution near the Earth's surface. We therefore expect all three techniques to contribute to 
future understanding of global-scale redistribution of surface mass.

Acknowledgements. We thank the IGS analysis center at Scripps Institution of Oceanography for freely providing GPS SINEX files for use by the geodetic community. The work of one of the authors (RSG) described in this paper was performed at the Jet Propulsion Laboratory, California Institute of Technology, under contract with the National Aeronautics and Space Administration. Support for the work of RSG was provided by the Earth Surface and Interior Focus Area of NASA. Support for GB was provided by NASA grants NNG04G099G and NAG5-13683. PJC acknowledges grant NER/A/S/2001/01166 from the UK Natural Environment Research Council, and DAL was supported by a Royal Society University Research Fellowship.

\section{References}

Bettadpur, S. (2007). UTCSR level-2 processing standards document for level-2 product release 0004. GRACE 327-742, CSR-GR-03-03, 17 pp., Center for Space Research, Univ. Texas, Austin.

Blewitt, G., and P. Clarke (2003). Inversion of Earth's changing shape to weigh sea level in static equilibrium with surface mass redistribution. J. Geophys. Res., 108(B6), 2311, doi:10.1029/2002JB002290.

Chao, B.F., and R.S. Gross (1987). Changes in the Earth's rotation and low-degree gravitational field induced by earthquakes. Geophys. J. R. astr. Soc., 91, pp. 569-596.

Cheng, M.K., and J. Ries (2007). Monthly estimates of C20 from 5 SLR satellites. GRACE Technical Note 05, 2 pp., Center for Space Research, Univ. Texas, Austin.

Cheng, M.K., and B.D. Tapley (2004). Variations in the Earth's oblateness during the past 28 years. J. Geophys. Res., 109, B09402, doi:10.1029/2004JB003028.

Clarke, P.J., D.A. Lavallée, G. Blewitt, and T. van Dam (2007). Basis functions for the consistent and accurate representation of surface mass loading. Geophys. J. Int., in press, doi:10.1111/j.1365-246X.2007.03493.x.

Desai, S.D. (2002). Observing the pole tide with satellite altimetry. J. Geophys. Res., 107(C11), 3186, doi:10.1029/2001JC001224.

Greatbatch, R.J. (1994). A note on the representation of steric sea level in models that conserve volume rather than mass. J. Geophys. Res., 99, pp. 12767-12771.

Gross, R.S. (2003). The GGFC Special Bureau for the Oceans: Past progress and future plans. In: Proceedings of the IERS Workshop on Combination Research and Global Geophysical Fluids. Richter, B., W. Schwegmann, and W.R. Dick (eds). pp. 131-138, IERS Technical Note No. 30, Bundesamts für Kartographie und Geodäsie, Frankfurt, Germany.
Gross, R.S. (2006). Combinations of Earth orientation measurements: SPACE2005, COMB2005, and POLE2005. Jet Propulsion Laboratory Publ. 06-3, 26 pp., Pasadena, Calif.

Gross, R.S., B.F. Chao, and S. Desai (1997). Effect of long-period ocean tides on the Earth's polar motion. Prog. Oceanogr., 40, pp. 385-397.

Gross, R.S., G. Blewitt, P. Clarke, and D. Lavallee (2004). Degree-2 harmonics of the Earth's mass load estimated from GPS and Earth rotation data. Geophys. Res. Lett., 31, L07601, doi:10.1029/2004GL019589.

Gross, R.S., I. Fukumori, and D. Menemenlis (2005). Atmospheric and oceanic excitation of decadal-scale Earth orientation variations. J. Geophys. Res., 110, B09405, doi:10.1029/2004JB003565.

Kalnay, E., M. Kanamitsu, R. Kistler, W. Collins, D. Deaven, L. Gandin, M. Iredell, S. Saha, G. White, J. Woollen, Y. Zhu, M. Chelliah, W. Ebisuzaki, W. Higgins, J. Janowiak, K.C. Mo, C. Ropelewski, J. Wang, A. Leetmaa, R. Reynolds, R. Jenne, and D. Joseph (1996). The NCEP/NCAR 40-year reanalysis project. Bull. Amer. Met. Soc. 77, pp. 437-471.

Kantha, L.H., J.S. Stewart, and S.D. Desai (1998). Long-period lunar fortnightly and monthly ocean tides. J. Geophys. Res., 103, pp. 12639-12647.

Kaula, W.M. (1966). Theory of Satellite Geodesy. Blaisdell, Waltham, Mass.

Milly, P.C.D., and A.B. Shmakin (2002). Global modeling of land water and energy balances. Part I: The Land Dynamics (LaD) model. J. Hydrometeor., 3(3), pp. 283-299.

Salstein, D.A. (2003). The GGFC Special Bureau for the Atmosphere of the International Earth Rotation and Reference Systems Service. In: Proceedings of the IERS Workshop on Combination Research and Global Geophysical Fluids. Richter, B., W. Schwegmann, and W.R. Dick (eds). pp. 131-138, IERS Technical Note No. 30, Bundesamts für Kartographie und Geodäsie, Frankfurt, Germany.

Stammer, D., C. Wunsch, I. Fukumori, and J. Marshall (2002). State estimation improves prospects for ocean research. Eos Trans. Amer. Geophys. Union, 83(27), pp. 289-295.

Tapley, B.D., S. Bettadpur, M. Watkins, and C. Reigber (2004). The gravity recovery and climate experiment: Mission overview and early results. Geophys. Res. Lett., 31, L09607, doi:10.1029/2004GL019920,.

Yoder, C.F., J.G. Williams, and M.E. Parke (1981). Tidal variations of Earth rotation. J. Geophys. Res., 86, pp. 881-891.

Zhou Y.H., D.A. Salstein, and J.L. Chen (2006). Revised atmospheric excitation function series related to Earth variable rotation under consideration of surface topography. J. Geophys. Res., 111, D12108, doi:10.1029/ 2005JD006608. 